\title{
A meta-analysis of Italian and Estonian individuals shows an effect of common variants in $H M G C R$ on blood apoB levels
}

\author{
Martina Rosticci $\ddagger^{\ddagger 1}$, Natalia Pervjakova ${ }^{\ddagger 2,3,4}$, Marika Kaakinen ${ }^{\ddagger 4,5}$, Arrigo F Cicero ${ }^{1}$, Arne $\mathbf{P}$ \\ Feufer $^{6}$, Letizia Marullo7 ${ }^{7}$ Reedik Mägi ${ }^{2}$, Krista Fischer ${ }^{2}$, Longda Jiang ${ }^{4}$, Sergio D'Addato', \\ Elisabetta Rizzoli', Gianmichele Massimo ${ }^{8}$, Marina Giovannini ${ }^{1}$, Sabrina Angelini ${ }^{8}$, Patrizia \\ Hrelia $^{8}$, Chiara Scapoli $\$, 7$, Claudio Borghi ${ }^{\S, 1}$ \& Inga Prokopenko ${ }^{*, \$, 4}$ \\ ${ }^{1}$ Medicine \& Surgery Sciences Department, Alma Mater Studiorum University of Bologna, Bologna, Italy \\ ${ }^{2}$ Estonian Genome Center, Institute of Genomics, University of Tartu, Tartu, Estonia \\ ${ }^{3}$ Department of Biotechnology, Institute of Molecular \& Cell Biology, University of Tartu, Tartu, Estonia \\ ${ }^{4}$ Genomics of Common Disease, Division of Diabetes, Endocrinology \& Metabolism, Department of Medicine, Imperial College \\ London, London, UK \\ ${ }^{5}$ Pharmacology \& Therapeutics, Division of Experimental Medicine, Department of Medicine, Imperial College London, London, UK \\ ${ }^{6}$ Institute of Bioinformatics \& Systems Biology, Helmholtz Zentrum München, München, Germany \\ ${ }^{7}$ Department of Life Sciences \& Biotechnology, University of Ferrara, Ferrara, Italy \\ ${ }^{8}$ Department of Pharmacy \& Biotechnology, Alma Mater Studiorum University of Bologna, Italy \\ *Author for correspondence: Tel.: +44 0207594 6501; i.prokopenko@imperial.ac.uk \\ $\ddagger$ Co-first authors \\ $\S$ Co-last authors
}

Aim: The aim of the study was to explore the effects of variants at HMGCR-KIF6 loci on a range of cardiometabolic phenotypes. Methods: We analyzed the range of variants within Genetics in Brisighella Health Study and KIF6 genes using an additive genetic model on 18 cardiometabolic phenotypes in a sample of 1645 individuals from the Genetics in Brisighella Health Study and replicated in 10,662 individuals from the Estonian Genome Center University of Tartu. Results: We defined directly the effects of rs3846662:C>A at $H M G C R$ on apoB levels. The analysis also confirmed effects of on low-density lipoprotein-cholesterol and total cholesterol levels. Variants in KIF6 gene did not reveal any associations with cardiometabolic phenotypes. Conclusion: This study highlights effect of HMGCR locus on assay-determined apoB levels, an infrequent measure of blood lipids in large studies.

First draft submitted: 7 December 2017; Accepted for publication: 21 March 2018; Published online: 7 September 2018

Keywords: ApoB $\bullet$ blood lipids $\bullet$ cardiovascular disease $\bullet$ HMGCR • KIF6

Cardiovascular disease (CVD) encompasses a wide range of pathological conditions, including raised blood pressure, hypertension, ischemic and coronary heart disease, cerebrovascular disease and heart failure [1]. To date, CVD is the leading cause of mortality and morbidity globally with an estimated number of 17.5 million deaths per year in the world, projected to reach 23.3 million by $2030[2,3]$. Several lines of evidence from epidemiological and biochemical studies have widely established that high level of LDL-C, low concentration of HDL-C and high level of total cholesterol (TC) play a pivotal role for the development of CVD [4], and moreover can be considered as important factors in the neurodegenerative pathology. In particular, high levels of LDL-C and triglycerides are causally related to the risk of coronary artery disease (CAD) [5-7]. Apolipoproteins are molecules that may function as the regulator of lipoprotein metabolism through their maintenance of the structure of the lipoprotein particles [8]. Apolipoproteins are known to bind lipids to form lipoproteins such as LDL, HDL, VLDL and iIDL that play an important function in recognition of these complexes by other molecules' receptors. While the number and type of apolipoproteins vary between lipoproteins, the LDL metabolite is mostly attached to apoB [9,10]. ApoB molecules, mainly apoB-100, are particles responsible for a transport function for cholesterol and triglycerides in the blood stream [10]. It has been estimated that $90-95 \%$ of apoB molecules circulating in plasma provide a good measure 
of the total number of LDL particles, reflecting the atherogenicity of LDL-C [11-15]. The genetic background and predictive value of LDL-fractions such as apoB has so far only been investigated in a limited number of relatively small studies $[7,13,15]$.

Over the past decade, more than 40 loci in human genome have been described with respect to the differential effect of statins on decreasing the risk of clinical end points including cardiovascular death and myocardial infarction, and on modulating lipid levels [16-19]. One of the most well-known genes in this respect is $H M G C R$, also known as $H M G-C o A$ reductase. $H M G C R$ is a transmembrane glycoprotein of the endoplasmatic reticulum that serves as a rate-limiting enzyme in cholesterol biosynthetic pathway [20,21]. Statins, the most used lipid-lowering drugs worldwide, act as HMG-CoA inhibitors, by actively occupying binding sites and thus blocking the association of enzyme with substrate [22]. Despite the fact that this mechanism of action proved its effectiveness in reducing the risk of CVD event and overall inflammation, there is a wide range of inter-individual variability to statin response [23]. This variation could be caused by alternative splicing occurring in exon 13 and other single nucleotide polymorphisms (SNP) located outside of HMGCR that contributes to differences in LDL cholesterol level [24].

KIF6 is a relatively recent candidate for CVD [25-27], initially suggested as contributing to cardiovascular risk within the European population [28]. KIF6 protein is a homodimeric molecule belonging to the KIF9 family of kinesins and is known to be ubiquitously expressed in the coronary arteries and vascular cells [29]. The function of KIF6 gene is yet poorly understood, although it is reported to mediate intracellular transport of organelles, protein complexes and mRNAs [30]. Variants at KIF6 gene are characterized by controversial observations. Specifically, a common SNP, rs20455 (c.2104:T>C; p[Trp719Arg]) resulting in a missense tryptophan to arginine substitution at position 719 of the codified polypeptide chain has been widely studied since 2007 [27,31,32]. In a meta-analysis of eight prospective studies, it has been demonstrated that carriers of the KIF6 719Arg variant were at an increased risk to develop CVD [33] and received a significant benefit from the statin therapy compared with 719Trp wild-type individuals [32]. However, variants within the KIF6 locus have not been reported for their effects on lipids levels in genome-wide association studies (GWAS) [34], and their role in regulating statin therapy response is not defined.

In this study, we aimed to investigate the role of $H M G C R$ and $K I F 6$ variants in the body metabolism through analysis of association with cardiometabolic phenotypes, including 14 quantitative traits and four categorical outcomes in two European studies in up to 12,307 individuals.

\section{Materials \& methods}

\section{Study populations \& sample collection}

The Genetics in Brisighella Health Study (GBHS) is a prospective, population-based longitudinal epidemiological investigation of genetic factors influencing a range of cardiometabolic phenotypes involving 2939 randomly selected Caucasian individuals (1491 men and 1448 women), aged 14-84 years, free of cardiovascular disease at enrolment, resident in the northern Italian rural town of Brisighella. The study started in 1972 and it is still ongoing. The town of Brisighella was originally selected as the site for the study, given a homogeneous life-style of its residents, with a very low rate of migration. Subjects were clinically evaluated at baseline and every 4 years thereafter by collecting an extensive amount of clinical and laboratory data. For the purpose of this study, we selected 1645 subjects from the 2008/2012 GBHS population survey, with available blood samples [35-38]. The sample was selected in order to avoid direct familial connections between volunteers, such as parent-child or brother-sisters pairs. Only one individual from each pair was selected for further analyses.

The Estonian Genome Center University of Tartu (EGCUT) is a prospective, volunteer-based sample of the Estonian resident adult population (aged $\geq 18$ years). The current number of participants - close to 52,000 represents about $5 \%$ of the Estonian adult population, making it ideally suited to population-based studies. General practitioners and medical personnel in the special recruitment offices have recruited participants throughout the country. At baseline, the general practitioners performed the standardized health examination of the participants, who also donated blood samples and filled out a 16-module questionnaire on health-related topics such as lifestyle, diet and clinical diagnoses described in WHO ICD-10. In total, 10,662 individuals were selected with available blood samples according to the requested protocols to complement the GBHS population data. In analogy with GBHS data, only one individual from each pair of relatives was selected for the analyses.

All participants provided written informed consent. The Ethics Committee of Human Studies, University of Tartu, Estonia and Ethical Board of the University of Bologna approved the studies. All experiments in all cohorts were performed in accordance with relevant guidelines and regulations. 


\section{Phenotypes}

We analyzed 18 cardiometabolic phenotypes (Supplementary Table 1) which consisted of 14 quantitative traits and four binary outcomes, including Type 2 diabetes (T2D), coronary artery disease (CAD), hypertension and hypercholesterolemia. Four disease statuses were defined following the standard international guidelines by ICD-10 codes between I20 and I25, and between I60 and I71 [39-41].

Quantitative phenotypes included total cholesterol (TC), HDL-C, LDL-C, triglycerides (TG), apoA1, apoB, fasting glucose, body mass index (BMI), waist circumference, systolic and diastolic blood pressure (SBP, DBP), heart rate, serum uric acid (UR) and creatinine (CREA). Laboratory methods for the measurement of each quantitative trait are summarized in Supplementary Table 2. Following indication reported elsewhere, in the analysis of DNA variant effects on lipids, we excluded individuals undergoing lipid-lowering therapy, while for individuals undergoing anti-hypertension therapy we added $15 \mathrm{mmHg}$ to SBP and $10 \mathrm{mmHg}$ to DBP [34,42].

To check the assumption about normally distributed quantitative traits, we performed a Shapiro-Wilk test on all phenotypes. Since normality test significantly deviated from normal distribution $(\mathrm{p}<0.05)$ for all phenotypes, we applied an inverse normal transformation of residuals on all traits. Residuals for lipids and BMI were adjusted for sex, age and age ${ }^{2}$, while fasting glucose, CREA, UR were adjusted for sex and age only. Anthropometric traits, DBP, SBP and heart rate were adjusted for sex, age, age ${ }^{2}$ and BMI. Residuals for categorical phenotype were calculated as follows: T2D was adjusted for sex, age and BMI; CAD was adjusted for sex and age; hypercholesterolemia for sex, age and age $^{2}$; and hypertension for sex, age, age ${ }^{2}$ and BMI. Normality test and transformation of data were run using $\mathrm{R}$ software version 3.3.1 [43].

Correlation between phenotypes were calculated using the polycor package implemented in $\mathrm{R}$ software [43]. Hetcor function allowed to perform a Pearson product-moment correlation test between quantitative traits, a polyserial correlation test between quantitative-discrete traits and a polychoric correlation test between discretediscrete traits.

\section{Genotyping, quality control \& imputation}

Genomic DNA of GBHS samples was isolated from EDTA-anticoagulated whole blood using the QIAamp DNA Blood kit (Qiagen, Hilden, Germany) as recommended by the manufacturer. DNA samples were diluted and stored as $10 \mathrm{ng} / \mu \mathrm{l}$ aliquots at $-20^{\circ} \mathrm{C}$. Five SNPs were genotyped: three in the KIF6 gene on chromosome 6, including one missense variant rs20455 (c.2104:T>C; p[Trp719Arg]) and two highly correlated intronic variants; and two in the HMGCR gene on chromosome 5, including one upstream variant (rs3761740:C>A) and another in intron 12. Linkage disequilibrium (LD) between the variants was calculated using Haploview software [44].

Genotyping was performed by real time using the $5^{\prime}$-nuclease allelic discrimination assay (TaqMan ${ }^{\circledR}$, Applied Biosystems, CA, USA), according to the manufacturer instruction. Negative controls were included to each reaction as a quality control.

Two independent sets containing 2589 and 8073 randomly selected individuals from EGCUT were used for replication. Samples were genotyped with commercially available Illumina Human370CNV and Illumina OMNIExpress arrays, respectively.

Sample and SNP quality control was undertaken within each contributing study. Sample quality control covered exclusions on the basis of call rate $(<95 \%)$; in the EGCUT additional exclusions were based on the extreme heterozygosity $( \pm 3 \mathrm{SD}$ ), sex discordance, cryptic relatedness (pihat $\geq 0.1$ ), and outlying ethnicity. SNP quality control covered exclusions on the basis of call rate $(<95 \%)$ across samples, minor allele frequency $(\mathrm{MAF} ;<1 \%)$ and extreme deviation from the Hardy-Weinberg equilibrium $\left(\mathrm{p}<1 \times 10^{-4}\right)$.

Within the EGCUT, the autosomal markers were imputed up to the 1000 Genomes project multiethnic reference panel (Phase III, March 2012 release). Imputation was performed using IMPUTEv2 software [45]. Poorly imputed variants (IMPUTE info <0.8) were excluded from further analysis.

\section{Association analysis}

For quantitative traits, we assumed an additive genetic model of effects and applied a linear regression and for categorical phenotypes - log-additive genetic effects and implemented a logistic regression. All single variant association analyses were run using R software [43] and PLINKv1.07 software in parallel [46,47]. Association analysis in two Estonian samples was run using SNPTESTv2.5 software using maximum likelihood genotype estimates on imputed SNP array data [48]. Meta-analysis of results from three studies was performed using GWAMA software [49]. Association tests were Bonferroni corrected for multiple testing, accounting for 19 non-highly $(\mathrm{r}<0.5)$ correlated 
phenotypes and three independent SNPs (the three genotyped KIF6 variants are highly correlated, $0.8<\mathrm{r}^{2}<1$ ) resulting in a study-wide significance threshold of $\mathrm{p}<8.7 \times 10^{-4}$ assuming the Bonferroni correction for multiple testing.

\section{Results}

We performed the association analysis of two HMGCR and three KIFG variants and 18 cardiometabolic phenotypes. Genotyping data for these five SNPs (Supplementary Table 3) were available in up to 12,307 individuals of European descent from two studies, including discovery performed in GBHS and replication undertaken in the EGCUT. All five SNPs passed quality control measures, and were then tested for association in each study and combined across the two studies by a fixed-effect meta-analysis.

Prior the analysis, we calculated linkage disequilibrium (LD) between two sets of genotyped SNPs rs3761740:C > A and rs3846662:C > A for HMGCR and rs20455 (c.2104:T>C; p.[Trp.719Arg]), rs9462535:A >C and rs9471077:G > A for KIFG tested in this study for the GBHS. The two variants within the HMGCR locus are only mildly correlated (Supplementary Figure $2 \mathrm{~A}$ ) and showed slightly lower LD $\left(\mathrm{r}_{\mathrm{rs} 3761740-\mathrm{rs} 3846662}^{2}=0.15\right)$ than that reported for 1000 Genomes project data for Europeans $\left(\mathrm{r}_{\mathrm{rr} 3761740-\mathrm{r} 33846662}=0.22\right)$. The variants within the KIF6 gene,

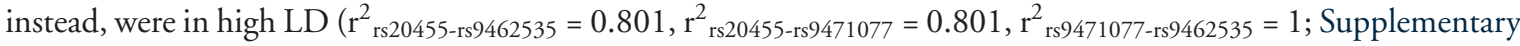
Figure $2 \mathrm{~B})$, consistent with LD patterns for Europeans from the 1000 Genomes project $\left(\mathrm{r}^{2}{ }_{\mathrm{rs} 20455-\mathrm{rs} 9462535}=0.86\right.$,

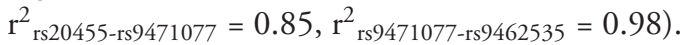

The power for our analysis was calculated using the Quanto software [50,51] given the different sample sizes for studied phenotypes, level of significance and minor allele frequency of the variants. The analysis revealed $80 \%$ power for $H M G C R$ variant rs3846662 (MAF $=0.45)$ at $\mathrm{p}=8.7 \times 10^{-4}$ to detect effects of $\beta>0.12$ for the smallest sample size $(\mathrm{n}=2400)$ and effect of $\beta>0.06$ for the maximum sample size $(\mathrm{n}=11,400)$ available for this variant. With the MAF $=0.35$ (KIF6 variants, $\left.\mathrm{MAF}_{\mathrm{rs} 20455}=0.365 ; \mathrm{MAF}_{\mathrm{rs} 96462535}=0.381 ; \mathrm{MAF}_{\mathrm{rs} 9471077}=0.384\right)$ we have about $80 \%$ power at $\mathrm{p}=8.7 \times 10^{-4}$ to detect effects of $\beta \geq 0.13$ for the smallest sample size $(\mathrm{n}=2400)$ and $\beta$ $\geq 0.06$ for the maximum sample size $(\mathrm{n}=11,409)$. With the $\mathrm{MAF}=0.10\left(H M G C R\right.$ variant $\left.\mathrm{MAF}_{\mathrm{rs} 3761740}=0.096\right)$ we have about $80 \%$ power at $\mathrm{p}=8.7 \times 10^{-4}$ to detect effects of $\beta>0.20$ for the smallest sample size $(\mathrm{n}=2400)$ and $\beta \geq 0.09$ for maximum sample size $(\mathrm{n}=11,400)$.

The discovery analysis in the GBHS confirmed the established effects of $H M G C R$ locus variant rs 3846662:C $>\mathrm{A}$ on LDL cholesterol levels $(\beta[\mathrm{SE}])=0.13[0.04], \mathrm{p}=0.001$; Supplementary Tables $1 \&$ 4). Meta-analysis of GBHS and EGCUT results confirmed the effect of the rs3846662:C>A on LDL cholesterol ( $\beta[S E]$ $\left.=0.13[0.03] ; \mathrm{p}=1.4 \times 10^{-5}\right)$. Our study reveals LDL cholesterol strong correlation with apoB levels $(\mathrm{r}=0.67)$ in our epidemiological data (Supplementary Figure 1), which also supports the finding of our analysis highlighting an effect of the previously suggested rs3846662:C $>\mathrm{A}$ variant at $H M G C R$ on assay-determined apoB levels in the combined GBHS and EGCUT analysis $\left(\beta[\mathrm{SE}]=0.07[0.02] ; \mathrm{p}=3.13 \times 10^{-5}\right.$; pmultiple testing corrected $\left.=8.7 \times 10^{-4}\right)$. The apoB level increasing allele was associated with increased LDL cholesterol, in accordance with previously reported epidemiological correlation [52], which confirms direct relationship of these two molecules. Meta-analysis also confirmed an association of the $H M G C R$ locus rs3846662: $\mathrm{C}>\mathrm{A}$ with TC $\left(\mathrm{p}=4.02 \times 10^{-5}\right)$. HMGCR rs 3761740:C $>$ A variant showed an additional associations with SBP $(\mathrm{p}=0.003)$ and DBP $(\mathrm{p}=0.007)$ in GBHS, but was not replicated in EGCUT and did not result in a significant overall effect in the meta-analysis of all the cohorts.

Association analysis of KIFG did not reveal any associations with cardiometabolic traits in the meta-analysis. The KIF6 rs20455 (c2104T>C; p.[Trp719Arg]) variant was associated with HDL-C ( $\mathrm{p}=0.001)$ and apoB levels (p $=0.008$ ) in GBHS but not in EGCUT (Table 1). The other two analyzed KIF6 variants (rs9471077:G $>\mathrm{A}$ and rs9462535:A $>C)$, both intronic, were associated with higher levels of TG $\left(p_{\text {rs } 9471077}=0.004 ; p_{\text {rs9462535 }}=0.003\right)$ and $\mathrm{apoB}\left(\mathrm{p}_{\mathrm{rs} 9471077}=0.001 ; \mathrm{p}_{\mathrm{rs} 9462535}=0.003\right)$, and lower HDL-C $\left(\mathrm{p}_{\mathrm{rs} 9471077}=0.009 ; \mathrm{p}_{\mathrm{rs} 9462535}=0.007\right)$ in GBHS but were not replicated in EGCUT (Table 1).

\section{Discussion}

We have explored the role of $H M G C R$ and $K I F 6$ variants in the body metabolism and response to statin treatment through analysis of association with cardiometabolic phenotypes in two European population-based studies in up to 12,307 individuals. In this study, we report a confirmation for association of $H M G C R$ locus variants with apoB levels. The HMGCR gene itself is a well-established locus associated with TC and LDL cholesterol levels. The first report about $H M G C R$ effects on cardiometabolic phenotypes was based on a comprehensive GWAS study 


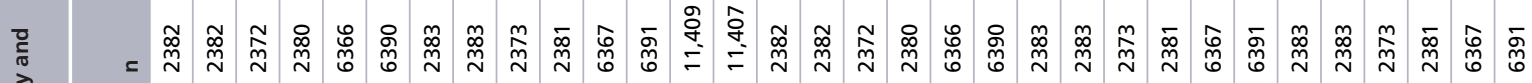

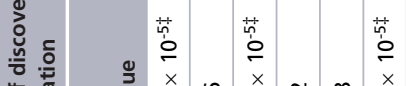

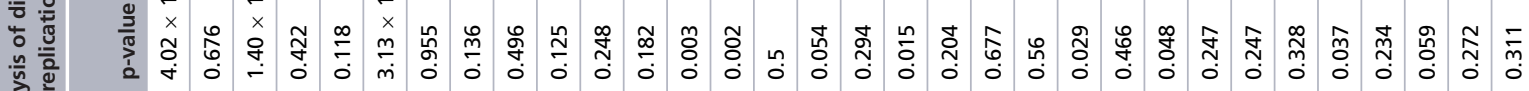

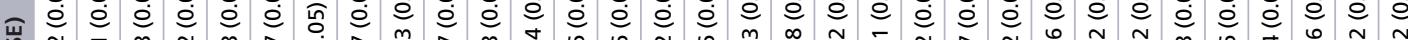

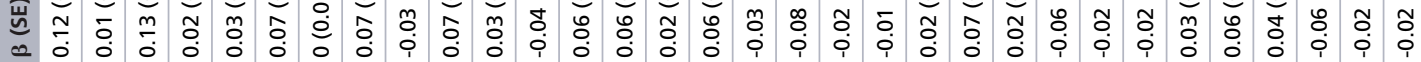

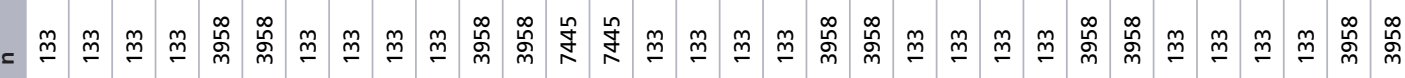

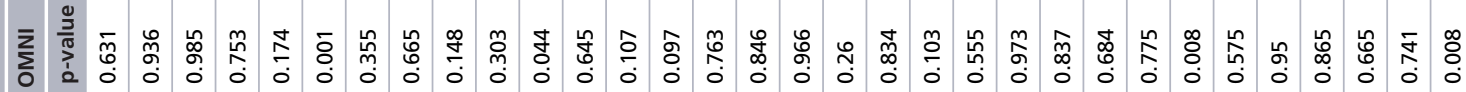

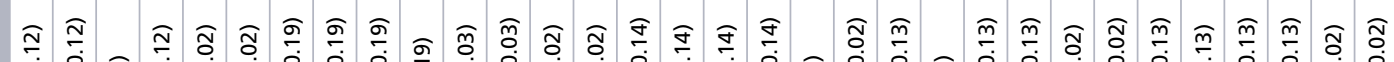

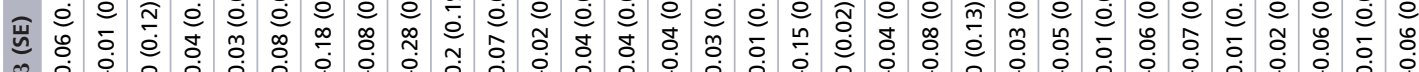

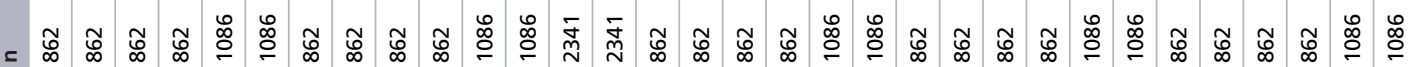

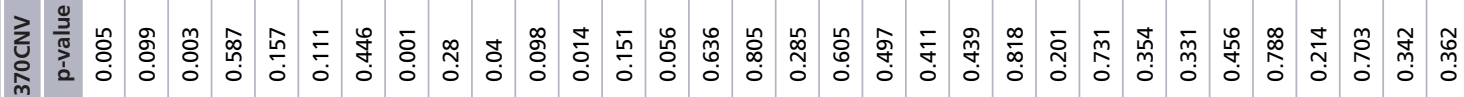

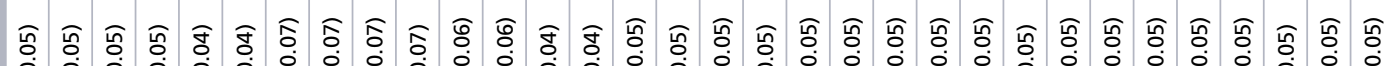

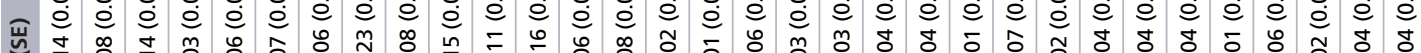

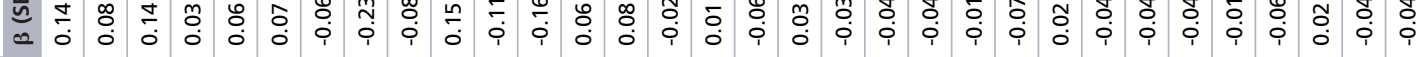

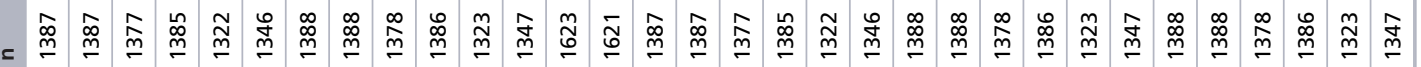

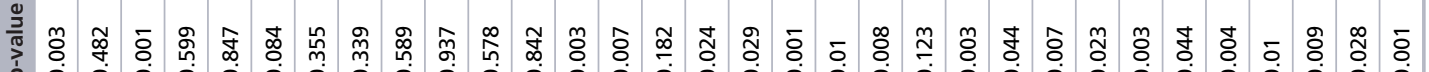

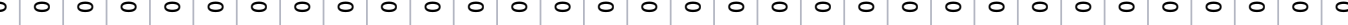

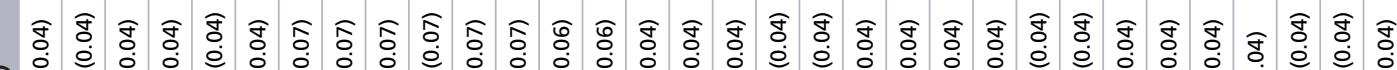

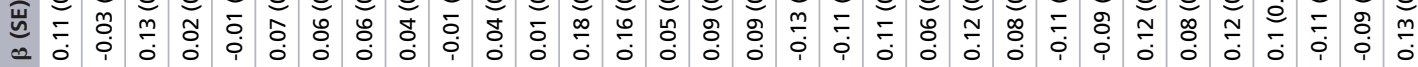

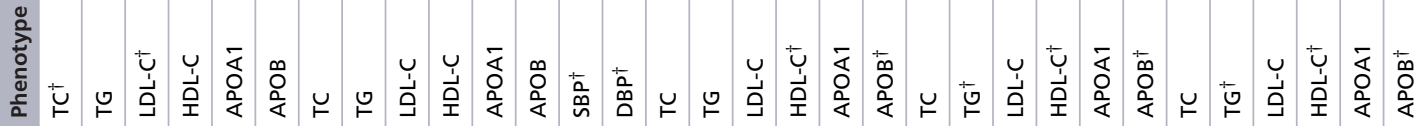

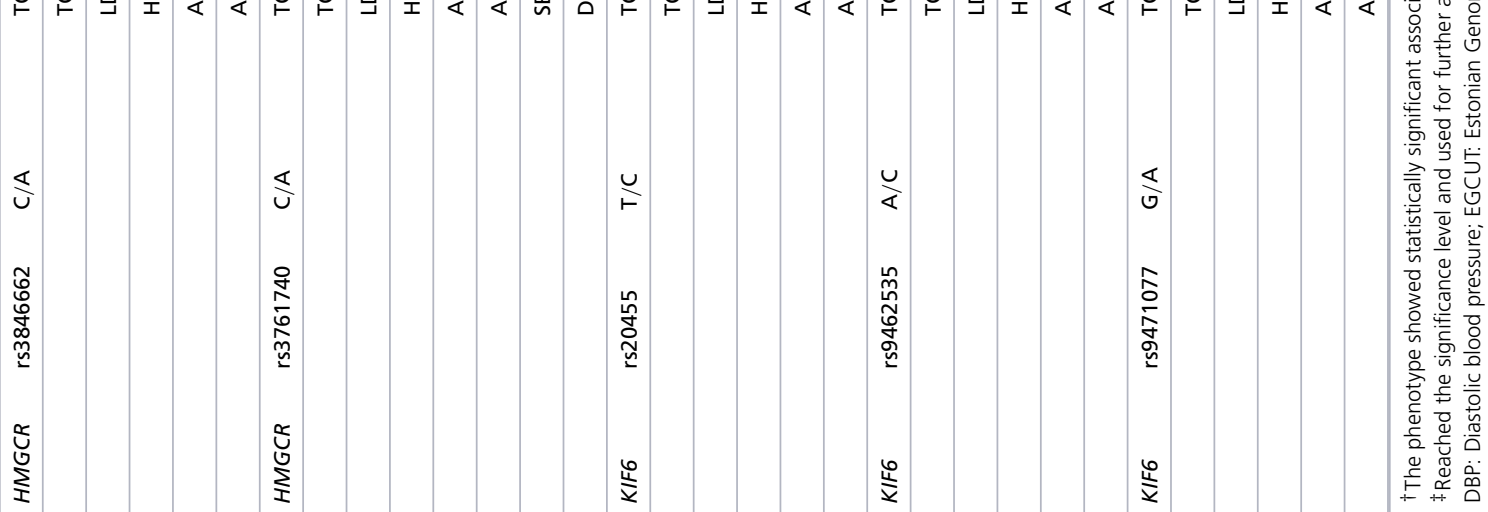


conducted in 16 population-based studies from up to 22,562 individuals [53]. This study reported rs3846662:C $>\mathrm{A}$ as a lead variant at $\operatorname{HMGCR}\left(\mathrm{p}=2.5 \times 10^{-19}\right.$ for TC; $\mathrm{p}=1.5 \times 10^{-11}$ for LDL cholesterol $)$ [53]. Another GWAS in more than 100,000 individuals of European ancestry, published a year later, also revealed a new association of genetic variants located in $H M G C R$ with coronary artery disease [54]. In parallel with this discovery, the SNP rs3846662 was for the first time suggested to be associated with apoB levels in the gene-centric analysis [55]. Moreover, additional evidence that pointed to the importance of this signal came from the study performed by Kettunen $e t$ al., where the downstream gene variant rs4703667, which is in high LD with intronic rs3846662, was also associated with the nuclear magnetic resonance-based metabolomics platform apoB levels [56]. Our study results are completely in line with previous reports and show the impact of the variant on assay-determined apoB levels in blood. The rs 3846662 variant located within intron 13 of HMGCR gene is not only associated with a modest increase of LDL cholesterol levels, but also modulates efficiency of $H M G C R$ pre-mRNA molecules by decreased level of naturally occurring nonfunctional transcripts [57]. This observation provides great support for our finding about the HMGCR locus association with apoB molecules that mainly transport LDL cholesterol molecules in human bloodstream.

ApoB levels are rarely measured within large-scale studies. Our study and association of the HMGCR genetic variant rs3846662: $\mathrm{C}>\mathrm{A}$ with apoB levels, thus bring a new highlight to the discussion of adding apoB to the standard clinical lipid measurements. It is important to note that our study has shown that the direction of genetic effect to apoB was similar to epidemiological correlation with LDL cholesterol, which proves the use of apoB molecule as a valuable predictor of severe CVD outcomes. In contrast to other major lipid groups, UK Biobank performed apoB measurement in hundreds of thousands of individuals to provide a better prediction of CVD risk and evaluate the efficacy of statin treatment. This hypothesis, if confirmed by further studies, may help in setting up novel approaches of diagnosis and treatment for hyperlipidemia and CVD. We believe that adding apoB to the standard clinical measurements would contribute to enhance the accuracy and outcome prediction of CVD and thus would help in choosing the right dose of a medication, resolving a problem of over-prescription of statins.

In our study, we did not observe any single-variant effect of the KIF6 locus on 18 cardiometabolic phenotypes. KIF6 has been reported in association with differential benefits, in terms of CVD events. For the first time, an association of genetic variants located in the KIF6 gene with coronary heart disease was demonstrated in a meta-analysis of seven prospective studies, including three prospective studies of individuals without coronary heart disease events at baseline and the placebo groups of four statin trials [58]. In particular, no association has been detected between the KIF6 p. Trp719Arg polymorphism and coronary heart disease in the Wellcome Trust Case Control Consortium [59]. It was suggested that the negative results may arise due to the case-control study design if statin therapy was not included in the selective criteria, therefore reducing the power of the study [58]. Another study that took into account statin therapy in 19 case-control studies comprised from a total of 17,000 cases and 39,369 controls also revealed no increased risk of nonfatal coronary artery disease by the p.Trp719Arg polymorphism [60]. In our study, we also did not find any significant associations between the three most studied polymorphisms at the KIF6 locus in terms of risk of CVD outcomes. The null findings from our study about the role of KIFG variants may be due to a lack of power, given the sample size, as the power analysis showed. Our analyses may also be biased by the type of data used for the analyses. Additionally, other studies have suggested that the KIFG gene may reveal unexpected associations due to chance, when studied in single cohorts [61]. Further studies, particularly based on meta-analysis of the literature and stratification for the type of statin would be better suited to overcome these limitations.

Our study also has some limitations. First, the sample sizes of our discovery and replication samples are relatively small, and further replication analyses in larger samples are required to confirm our novel findings. Moreover, the biological validity and clinical usefulness of the obtained results has to be verified using functional approaches [62]. Functional validation might involve multiple independent siRNA duplexes to validate the set of genes affecting apoB secretion distinguished from the effect caused by cell viability [62].

Finally, the set of cardiometabolic diseases used in our analysis has been chosen arbitrarily, even if the diagnostic cut-offs applied are those suggested by the main specific international guidelines. We expect that further comprehensive multiphenotype analyses might improve our understanding of the effects of specific loci on groups of related traits, thus to dissect better their pathophysiological impact on human health. Beyond that, the data are reliable and obtained from two European population-based studies, well characterized and defined [63,64]. 


\section{Conclusion}

In conclusion, this study did not reveal any association between the $K I F 6$ and cardiometabolic traits, but highlighted a novel effect of $H M G C R$ variants on apoB levels, which might be predictive of CVD risk. Further investigation of apoB levels variability in relation to CVD would be important. Additionally, evaluation of biological mechanisms underlying this relationship between the HMGCR gene and lipids metabolism will facilitate the advance of our knowledge and help in implementation of findings from this study in more personalized approaches for CVD prevention and therapy.

\section{Summary points}

- Cardiovascular diseases (CVDs) are the number one cause of death globally. According to the WHO data, an estimated 17.7 million people died from CVDs in 2015, representing $31 \%$ of all global deaths.

- Lipoproteins, such as LDL-C, HDL-C and total cholesterol, play a pivotal role for the development of CVD.

- We conducted the meta-analysis for cardiometabolic phenotypes, including 14 quantitative traits and four categorical traits, in two European studies in up to 12,307 individuals.

- Meta-analysis of Genetics in Brisighella Health Study and Estonian Genome Center University of Tartu results confirmed the effect of the variant rs3846662 on LDL cholesterol.

- The epidemiological data reveals LDL cholesterol strong correlation with apoB molecule.

- Meta-analysis confirmed previously suggested effect of the variant rs3846662 to apoB molecule.

- Association analysis of KIF6 variants did not reveal any associations with cardiometabolic traits in the meta-analysis.

- The variant rs3761740 in HMGCR gene showed an associations with systolic and diastolic blood pressure in Genetics in Brisighella Health Study, but not in Estonian Genome Center University of Tartu.

\section{Supplementary data}

To view the supplementary data that accompany this paper please visit the journal website at: https://www.futuremedicine.com/doi/suppl/10.2217/bmm-2017-0431

\section{Author contributions}

M Rosticci, L Marullo, C Borghi and I Prokopenko conceived and designed the experiments. M Rosticci, N Pervjakova, M Kaakinen, L Marullo, L Jiang, S D'Addato, C Borghi and I Prokopenko performed the experiments. M Rosticci, N Pervjakova, AF Cicero, R Mägi, K Fischer and C Borghi contributed data. N Pervjakova, M Kaakinen, L Marullo, AP feufer, M Rosticci, AF Cicero, R Mägi, K Fischer, L Jiang, S D’Addato, E Rizzoli, G Massimo, M Giovannini, S Angelini, P Hrelia, C Scapoli, C Borghi and I Prokopenko wrote the paper. R Mägi, C Scapoli, Borghi and I Prokopenko supervised the work.

\section{Acknowledgments}

The authors acknowledge High Performance Computing Center of University of Tartu and the Imperial College Research Computing Service, doi:10.14469/hpc/2232.

\section{Financial \& competing interests disclosure}

This study was supported by European Union H2020 grants 692145 (ePerMed I/II), 676550, 654248; Estonian Research Council grants IUT20-60, and PerMed I, NIASC, EIT - Health and European Union through the European Regional Development Fund (project no. 2014-2020.4.01.15-0012 GENTRANSMED); European Commission under the Marie Curie Intra-European Fellowship (project MARVEL (PIEF-GA-2013-P48951); the German National Research Ministry BMBF 01EZ0874, the Elsie Widdowson Fellowship, the Wellcome Trust Seed Award in Science WT205915, the World Cancer Research Fund (WCRF UK) and World Cancer Research Fund International (2017/1641), the European Union's Horizon 2020 research and innovation programme (DYNAhealth, H2020-PHC-2014-633595). The 2008 survey of the Brisighella Heart Study was supported by unrestricted grants from the Fondazione del Monte e and from Carisbo Foundation (Private Banks Foundations). The authors have no other relevant affiliations or financial involvement with any organization or entity with a financial interest in or financial conflict with the subject matter or materials discussed in the manuscript apart from those disclosed.

No writing assistance was utilized in the production of this manuscript. 


\section{Ethical conduct of research}

The authors state that they have obtained appropriate institutional review board approval or have followed the principles outlined in the Declaration of Helsinki for all human or animal experimental investigations. In addition, for investigations involving human subjects, informed consent has been obtained from the participants involved.

\section{Open access}

This work is licensed under the Attribution-NonCommercial-NoDerivatives 4.0 Unported License. To view a copy of this license, visit http://creativecommons.org/licenses/by-nc-nd/4.0/

\section{References}

Papers of special note have been highlighted as: $\bullet$ of interest; $\bullet \bullet$ of considerable interest

1. Taylor F, Ward K, Moore TH et al. Statins for the primary prevention of cardiovascular disease. Cochrane Database Syst. Rev. 31(1), CD004816 (2013).

2. Phan BAP, Bittner V. Lipid-lowering therapy in patients 75 years and older: clinical priority or superfluous therapy? Prog. Cardiovasc. Dis. 57(2), 187-196 (2014).

3. Global atlas on cardiovascular disease prevention and control. In: Global Atlas on Cardiovascular Disease Prevention and Control, Policies, Strategies and Interventions. WHO, World Heart Federation, World Stroke Organization, Geneva, Switzerland, 164 (2011).

4. Bays HE, Patel MD, Mavros P et al. Real-world data to assess changes in low-density lipoprotein cholesterol and predicted cardiovascular risk after ezetimibe discontinuation post reporting of the Ezetimibe and Simvastatin in Hypercholesterolemia Enhances Atherosclerosis Regression trial. J. Clin. Lipidol. 11(4), 929-937 (2017).

5. Stamler J, Daviglus ML, Garside DB, Dyer AR, Greenland P, Neaton JD. Relationship of baseline serum cholesterol levels in 3 large cohorts of younger men to long-term coronary, cardiovascular, and all-cause mortality and to longevity. JAMA 284(3), 311-318 (2000).

-• Shows the strategic importance of population-wide primary prevention of unfavorable serum cholesterol levels.

6. Do R, Willer CJ, Schmidt EM et al. Common variants associated with plasma triglycerides and risk for coronary artery disease. Nat. Genet. 45, 1345-1352 (2013).

7. Tani S, Yagi T, Atsumi W, Kawauchi K, Matsuo R, Hirayama A. Relation between low-density lipoprotein cholesterol/apolipoprotein B ratio and triglyceride-rich lipoproteins in patients with coronary artery disease and Type 2 diabetes mellitus: a cross-sectional study. Cardiovasc. Diabetol. 16(1), 123 (2017).

8. Mahley RW, Innerarity TL, Rall SC, Weisgraber KH. Plasma lipoproteins: apolipoprotein structure and function. J. Lipid Res. 25(12), 1277-1294 (1984).

9. Gotto AM. High-density lipoproteins: Biochemical and metabolic factors. Am. J. Cardiol. 52(4), B2-B4 (1983).

10. Sacks FM. The crucial roles of apolipoproteins $\mathrm{E}$ and C-III in apoB lipoprotein metabolism in normolipidemia and hypertriglyceridemia. Curr. Opin. Lipidol. 26(1), 56-63 (2015).

11. Lamarche B, Tchernof A, Moorjani S et al. Small, dense low-density lipoprotein particles as a predictor of the risk of ischemic heart disease in men. Prospective results from the Québec Cardiovascular Study. Circulation 95(1), 69-75 (1997).

12. Lamarche B, Moorjani S, Lupien PJ et al. Apolipoprotein A-I and B levels and the risk of ischemic heart disease during a five-year follow-up of men in the Quebec cardiovascular study. Circulation 94(3), 273-278 (1996).

-. The prospective study shows the importance of apolipoprotein $B$ as a risk factor for ischemic heart disease.

13. St-Pierre AC, Ruel IL, Cantin B et al. Comparison of various electrophoretic characteristics of LDL particles and their relationship to the risk of ischemic heart disease. Circulation 104(19), 2295-2299 (2001).

14. Linton MF, Yancey PG, Davies SS, Jerome WGJ, Linton EF, Vickers KC. The Role of Lipids and Lipoproteins in Atherosclerosis. De Groot LJ, Chrousos G, Dungan K (Eds). South Dartmouth, MA, USA (2015).

15. Schaefer EJ, Tsunoda F, Diffenderfer M, Polisecki E, Thai N, Asztalos B. The measurement of lipids, lipoproteins, apolipoproteins, fatty acids, and sterols, and next generation sequencing for the diagnosis and treatment of lipid disorders. Endotext (1), 1-28 (2016).

16. Poduri A, Khullar M, Bahl A, Sehrawat BS, Sharma Y, Talwar KK. Common variants of HMGCR, CETP, APOAI, ABCB1, CYP3A4, and CYP7A1 genes as predictors of lipid-lowering response to atorvastatin therapy. DNA Cell Biol. 29, 629-637 (2010).

- Shows that variants located in 3-hydroxy-3-methylglutaryl-CoA reductase gene can be used as predictors of lipid-lowering response to atorvastatin therapy.

17. Polisecki E, Peter I, Simon JS et al. Genetic variation at the NPC1L1 gene locus, plasma lipoproteins, and heart disease risk in the elderly. J. Lipid Res. 51, 1201-1207 (2010).

18. Hopewell JC, Parish S, Offer A et al. Impact of common genetic variation on response to simvastatin therapy among 18705 participants in the Heart Protection Study. Eur. Heart J. 34, 982-992 (2013). 
19. Chasman DI, Giulianini F, MacFadyen J, Barratt BJ, Nyberg F, Ridker PM. Genetic determinants of statin-induced low-density lipoprotein cholesterol reduction: the justification for the use of statins in prevention: an intervention trial evaluating rosuvastatin (JUPITER) trial. Circ. Cardiovasc. Genet. 5, 257-264 (2012).

20. Goldstein JL, Brown MS. Regulation of the mevalonate pathway. Nature 343, 425-430 (1990).

21. Arouche-Delaperche L, Allenbach Y, Amelin D et al. Pathogenic role of anti-signal recognition protein and anti-3-hydroxy-3-methylglutaryl-CoA reductase antibodies in necrotizing myopathies: myofiber atrophy and impairment of muscle regeneration in necrotizing autoimmune myopathies. Ann. Neurol. 81(4), 538-548 (2017).

22. Istvan ES. Structural mechanism for statin inhibition of HMG-CoA reductase. Science292(5519), 1160-1164 (2001).

23. Medina MW, Krauss RM. The role of HMGCR alternative splicing in statin efficacy. Trends Cardiovasc. Med. 19(5), $173-177$ (2009).

24. Yu CY, Theusch E, Lo K et al. HNRNPA1 regulates HMGCR alternative splicing and modulates cellular cholesterol metabolism. Hum. Mol. Genet. 23(2), 319-332 (2014).

25. Hu M, Lui SSH, Mak VWL et al. Pharmacogenetic analysis of lipid responses to rosuvastatin in Chinese patients. Pharmacogenet. Genomics 20(10), 634-637 (2010).

26. Hamidizadeh L, Haji HB, Babaee BMA, Dastsooz H, Khazaei NA, Fardaei M. Impact of KIF6 polymorphism Rs20455 on coronary heart disease risk and effectiveness of statin therapy in 100 patients from Southern Iran. Arch. Iran Med. 18(10), 683-687 (2015).

27. Arsenault BJ, Boekholdt SM, Hovingh GK et al. The 719Arg variant of KIF6 and cardiovascular outcomes in statin-treated, stable coronary patients of the treating to new targets and incremental decrease in end points through aggressive lipid-lowering prospective studies. Circ. Cardiovasc. Genet. 5(1), 51-57 (2012).

28. Bare LA, Morrison AC, Rowland CM et al. Five common gene variants identify elevated genetic risk for coronary heart disease. Genet. Med. 9, 682-689 (2007).

29. Miki H, Okada Y, Hirokawa N. Analysis of the kinesin superfamily: insights into structure and function. Trends Cell Biol. 15(9), $467-476$ (2005).

30. Li Y, Iakoubova OA, Shiffman D, Devlin JJ, Forrester JS, Superko HR. KIF6 polymorphism as a predictor of risk of coronary events and of clinical event reduction by statin therapy. Am. J. Cardiol. 106(7), 994-998 (2010).

31. Iakoubova OA, Tong CH, Rowland CM et al. Association of the trp719arg polymorphism in kinesin-like protein 6 with myocardial infarction and coronary heart disease in 2 prospective trials. The CARE and WOSCOPS Trials. J. Am. Coll. Cardiol. 51, 435-443 (2008).

32. Iakoubova OA, Robertson M, Tong $\mathrm{CH}$ et al. KIF6 Trp719Arg polymorphism and the effect of statin therapy in elderly patients: results from the PROSPER study. Eur. J. Cardiovasc. Prev. Rehabil. 17, 455-461 (2010).

33. Peng P, Lian J, Huang RS et al. Meta-analyses of KIF6 Trp719Arg in coronary heart disease and statin therapeutic effect. PLoS ONE 7(12) (2012).

- Demonstrates the effect of Trp719Arg of kinesin family member 6 gene in the risk of coronary heart disease in individuals of European ancestry.

34. Willer CJ, Schmidt EM, Sengupta S et al. Discovery and refinement of loci associated with lipid levels. Nat. Genet. 45, 1274-1283 (2013).

35. Cicero AF, Rosticci M, Bove $\mathrm{M}$ et al. Serum uric acid change and modification of blood pressure and fasting plasma glucose in an overall healthy population sample: data from the Brisighella Heart Study. Ann. Med. 49(4), 275-282 (2017).

36. Cicero AFG, Rosticci M, Fogacci F, Grandi E, D’Addato S, Borghi C. High serum uric acid is associated to poorly controlled blood pressure and higher arterial stiffness in hypertensive subjects. Eur. J. Intern. Med. 37(1)), 38-42 (2017).

37. Cicero AFG, Salvi P, D’Addato S, Rosticci M, Borghi C. Association between serum uric acid, hypertension, vascular stiffness and subclinical atherosclerosis: data from the brisighella heart study. J. Hypertens. 32(1), 57-64 (2014).

38. Cicero AFG, D'Addato S, Santi F, Ferroni A, Borghi C. Leisure-time physical activity and cardiovascular disease mortality: the Brisighella Heart Study. J. Cardiovasc. Med. 13(9), 559-564 (2012).

39. Rydén L, Grant PJ, Anker SD et al. ESC guidelines on diabetes, pre-diabetes, and cardiovascular diseases developed in collaboration with the EASD - summary. Diab. Vasc. Dis. Res. 11(3), 133-173 (2014).

40. Thygesen K, Alpert JS, Jaffe AS et al. Third universal definition of myocardial infarction. Eur. Heart J. 33, 2551-2567 (2012).

41. Mancia G, Fagard R, Narkiewicz K et al. 2013 ESH/ESC practice guidelines for the management of arterial hypertension. Blood Press. 23(1), 3-16 (2014).

42. The International Consortium for Blood Pressure Genome-Wide Association Studies. Genetic variants in novel pathways influence blood pressure and cardiovascular disease risk. Nature 478, 103-109 (2011).

43. R Core Team. R: a language and environment for statistical computing (2014). www.r-project.org/

44. Barrett JC, Fry B, Maller J, Daly MJ. Haploview: analysis and visualization of LD and haplotype maps. Bioinformatics 21, 263-265 (2005).

45. Howie BN, Donnelly P, Marchini J. A flexible and accurate genotype imputation method for the next generation of genome-wide association studies. PLoS Genet. 5(6), e1000529 (2009). 
46. Purcell S. PLINK v1.07 (10-Oct-2009). www.cog-genomics.org/plink2

47. Purcell S, Neale B, Todd-Brown K et al. PLINK: a tool set for whole-genome association and population-based linkage analyses. Am. J. Hum. Genet. 81, 559-575 (2007).

48. Marchini J, Howie B, Myers S, McVean G, Donnelly P. A new multipoint method for genome-wide association studies by imputation of genotypes. Nat. Genet. 39, 906-913 (2007).

49. Mägi R, Morris AP. GWAMA: software for genome-wide association meta-analysis. BMC Bioinformatics 11, 288 (2010).

50. Gauderman WJ. Sample size requirements for matched case-control studies of gene-environment interaction. Stat. Med. 21(1), 35-50 (2002).

51. Gauderman WJ. Sample size requirements for association studies of gene-gene interaction. Am. J. Epidemiol. 155(5), 478-484 (2002).

52. Lu M, Lu Q, Zhang Y, Tian G. ApoB/apoA1 is an effective predictor of coronary heart disease risk in overweight and obesity. J. Biomed. Res. 25(4), 266-273 (2011).

53. Aulchenko YS, Ripatti S, Lindqvist I et al. Loci influencing lipid levels and coronary heart disease risk in 16 European population cohorts. Nat. Genet. 41(1), 47-55 (2009).

54. Teslovich TM, Musunuru K, Smith AV et al. Biological, clinical and population relevance of 95 loci for blood lipids. Nature 466, 707-713 (2010).

55. Talmud PJ, Drenos F, Shah S et al. Gene-centric association signals for lipids and apolipoproteins identified via the humanCVD beadchip. Am. J. Hum. Genet. 85(5), 628-642 (2009).

56. Kettunen J, Demirkan A, Würtz P et al. Genome-wide study for circulating metabolites identifies 62 loci and reveals novel systemic effects of LPA. Nat. Commun. 7, 11122 (2016).

57. Burkhardt R, Kenny EE, Lowe JK et al. Common SNPs in HMGCR in Micronesians and Whites associated with LDL cholesterol levels affect alternative splicing of exon13. Arterioscler. Thromb. Vasc. Biol. 28(11), 2078-2084 (2008).

58. Li Y, Iakoubova OA, Shiffman D, Devlin JJ, Forrester JS, Superko HR. KIFG polymorphism as a predictor of risk of coronary events and of clinical event reduction by statin therapy. Am. J. Cardiol. 106(7), 994-998 (2010).

59. Wellcome Trust Case Control Consortium. Genome-wide association study of 14,000 cases of seven common diseases and 3000 shared controls. Nature 447(7145), 661-678 (2007).

60. Assimes TL, Hlm H, Kathiresan S et al. Lack of association between the Trp719Arg polymorphism in kinesin-like protein-6 and coronary artery disease in 19 case-control studies. J. Am. Coll. Cardiol. 56(19), 1552-1563 (2010).

61. Wu G, Li G Bin, Dai B. Association of KIFG variant with lipid level and angiographic coronary artery disease events risk in the Han Chinese population. Molecules 17, 11269-11280 (2012).

62. Shen X, Wang W, Wang L et al. Identification of genes affecting apolipoprotein B secretion following siRNA-mediated gene knockdown in primary human hepatocytes. Atherosclerosis 222, 154-157 (2012).

63. Mueller JC, Lõhmussaar E, Mägi R et al. Linkage disequilibrium patterns and tagSNP transferability among European populations. Am. J. Hum. Genet. 76, 387-398 (2005).

64. Cicero AFG, Salvi P, D’Addato S, Rosticci M, Borghi C. Association between serum uric acid, hypertension, vascular stiffness and subclinical atherosclerosis: data from the Brisighella Heart Study. J. Hypertens. 32, 57-64 (2014). 